Artículo original

\title{
Trastornos gastrointestinales funcionales después de un episodio de dengue no grave sin signos de alarma
}

\author{
Carlos Alberto Velasco-Benítez, Claudia Jimena Ortiz-Rivera
}

Departamento de Pediatría, Universidad del Valle, Cali, Colombia

Introducción. La patogenia de los trastornos funcionales gastrointestinales involucra agentes infecciosos como los virus.

Objetivo. Investigar el desarrollo de trastornos funcionales gastrointestinales en niños, a los 3, 6, 9 y 12 meses después de un episodio de dengue no grave sin signos de alarma. Materiales y métodos. Se hizo un estudio de cohorte de 73 niños con diagnóstico de dengue no grave sin signos de alarma atendidos en el Hospital Universitario del Valle "Evaristo García" y de 62 niños sanos de Cali, Colombia. Mediante el 'Cuestionario para síntomas gastrointestinales pediátricos Roma III' (Questionnaire for Pediatric Gastrointestinal Symptoms Rome III, QPGS-III), se determinaron los trastornos gastrointestinales funcionales a los 3, 6, 9 y 12 meses de seguimiento después de un episodio de dengue no grave sin signos de alarma. Se calcularon las medidas de tendencia central, riesgo relativo y prueba de ji al cuadrado, y se usó la prueba exacta de Fisher con un nivel de significación $(p)$ menor de 0,05.

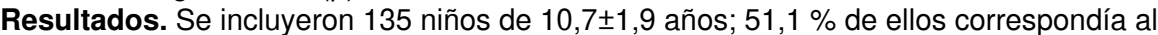
sexo masculino y $19,3 \%$ presentaba algún trastorno funcional gastrointestinal $(9,6 \%$ con dolor abdominal relacionado). El riesgo de presentar algún trastorno funcional gastrointestinal con dolor abdominal relacionado a los 3, 6, 9 y 12 meses de seguimiento en niños con dengue no grave sin signos de alarma fue mayor que sin dicho antecedente, pero sin diferencias significativas.

Conclusión. Los resultados del estudio sugieren que el dengue no grave sin signos de alarma no incrementó el riesgo de trastornos gastrointestinales funcionales y dolor abdominal relacionado a lo largo de 12 meses de seguimiento.

Palabras clave: enfermedades gastrointestinales; dengue; niño; adolescente.

Post-infectious functional gastrointestinal disorders in children after a non-severe dengue episode without warning signs

Introduction: The pathogenesis of functional gastrointestinal disorders involves infectious agents such as viruses.

Objective: To study the development of functional gastrointestinal disorders 3, 6, 9 and 12 months after an episode of non-severe dengue without warning signs in children.

Materials and methods: We conducted a cohort study in 73 children diagnosed with non-severe dengue without warning signs at Hospital Universitario del Valle "Evaristo Garcia" and 62 healthy children from Cali, Colombia. Using the Questionnaire for Pediatric Gastrointestinal Symptoms Rome III (QPGS-III) in Spanish we identified functional gastrointestinal disorders 3, 6, 9, and 12 months after non-severe dengue without warning signs. Measurements of central tendency, relative risk, chi square, and Fisher's exact test were performed, with $p<0.05$ being significant.

Results: We included 135 children who were $10.7 \pm 1.9$ years old; $51.1 \%$ of them were male and $19.3 \%$ had a functional gastrointestinal disorder $(9.6 \%$ of them had abdominal pain related to functional gastrointestinal disorders). There was a greater risk to present a functional gastrointestinal disorder and related abdominal pain in children after non-severe dengue without warning signs at 3, 6, 9, and 12 months of follow-up, but without significant differences. Conclusion: Our study suggests that non-severe dengue without warning signs does not increase the risk of functional gastrointestinal disorders and related abdominal pain for up to 12 months of follow-up.

Keywords: Gastrointestinal diseases; dengue; child; adolescent.

Entre una tercera y una cuarta parte de los escolares y adolescentes colombianos presentan algún trastorno funcional gastrointestinal según los criterios de Roma III y en 10,4 a 11,1\% de ellos el dolor abdominal está relacionado con dichos trastornos $(1,2)$. La patogenia de estos trastornos se relaciona con múltiples factores, y en el modelo biopsicosocial de su fisiopatología, los agentes infecciosos desempeñan un papel (3). En un par de 
estudios de niños se ha demostrado que dichos trastornos pueden desarrollarse como secuela de una diarrea aguda bacteriana, viral o parasitaria $(4,5)$, aunque en otro estudio sobre diarrea viral no se pudo establecer dicha asociación (6).

El dengue es una enfermedad viral transmitida por el mosquito Aedes aegypti, cuya incidencia cíclica en Colombia y en el departamento del Valle del Cauca se ha venido incrementando en los últimos años; los niños menores de 15 años son los más frecuentemente afectados $(7,8)$. Según la Organización Mundial de la Salud (OMS), el dengue se clasifica como grave (síndrome de choque) o no grave (probable, sin signos de alarma o con ellos) (9). El diagnóstico del dengue no grave sin signos de alarma se hace mediante pruebas de laboratorio (IgM, NS1); el cuadro clínico incluye fiebre y por lo menos dos de los siguientes síntomas o signos: náuseas, vómitos, erupción, leucopenia, artralgia y mialgia, además del resultado positivo en la prueba del torniquete (9).

En un estudio observacional en 4.023 niños colombianos entre los 8 y los 18 años de edad, se encontró que $23,0 \%$ presentaba algún trastorno funcional gastrointestinal según los criterios de Roma III, que 7,5 \% había tenido dengue durante el año anterior, y que los escolares entre los 8 y 12 años de edad tenían 1,56 veces más oportunidad de presentar dengue en ese mismo periodo $\left(\mathrm{IC}_{95 \%} 1,20-2,04 ; \mathrm{p}=0,0006\right)$ (10). Por ello, el confirmar la presencia de trastornos funcionales gastrointestinales después de una infección de dengue no grave en niños podría constituir un hallazgo importante.

El objetivo del presente estudio fue estudiar el desarrollo de trastornos funcionales gastrointestinales en niños después de un episodio de dengue no grave a los 3, 6, 9 y 12 meses de seguimiento.

\section{Materiales y métodos}

Se hizo un estudio observacional descriptivo, no experimental, analítico, y de cohorte. De los niños que consultaron al servicio de urgencias del Hospital Universitario del Valle "Evaristo García" en Cali entre el 16 de febrero y el 15 de agosto de 2013, se incluyeron aquellos entre los 8 y los 14 años de edad con diagnóstico de dengue no grave, y se excluyeron los críticamente enfermos o con otras infecciones virales o bacterianas y aquellos con enfermedades como gastritis, enfermedad por reflujo gastroesofágico, estreñimiento orgánico, enfermedad inflamatoria intestinal o enfermedad celíaca.

Antes de participar en el estudio, los padres o tutores de los niños y los niños mismos firmaron un consentimiento informado.

En el grupo de expuestos con dengue no grave se incluyeron 73 niños. Posteriormente, cada caso índice fue emparejado con 62 niños sanos de una institución educativa pública de Cali, con edades similares y del mismo sexo. Los niños entre los 8 y los 10 años de edad respondieron el 'Cuestionario para síntomas gastrointestinales pediátricos Roma III para escolares y adolescentes' (QPGS-III) con la ayuda de uno de los investigadores, en tanto que los niños mayores de 10 años lo hicieron por ellos mismos, con el fin de determinar los trastornos funcionales gastrointestinales en cinco momentos: al ingresar al estudio, y a los 3, 6, 9 y 12 meses de seguimiento. EI QPGS-III ya ha sido validado, probado y utilizado en niños colombianos y latinoamericanos por nuestro grupo de investigación (11-15).

Se tomó el peso y la talla de los niños utilizando el programa AnthroPlus de la OMS y se clasificaron según el índice de masa corporal como: malnutridos (talla normal: entre -2 y +1 desviaciones estándar (DE); 
sobrepeso: entre +1 y $+2 \mathrm{DE}$; obeso: $\geq+3 \mathrm{DE}$; desnutrido: entre -2 y $-3 \mathrm{DE}$, y desnutrido grave: $\geq-3 \mathrm{DE}(16)$. Según la talla para la edad, se clasificaron como: de talla normal, entre -2 y $+3 \mathrm{DE}$; de talla alta, $\geq+3 \mathrm{DE}$; de talla baja, entre -2 y $-3 D E$, o de talla baja grave, $\geq-3 D E$.

El cálculo del tamaño de la muestra se basó en la información obtenida del estudio pediátrico sobre trastornos funcionales gastrointestinales después de una infección viral de Saps, et al. (6). El número mínimo de niños expuestos con dengue no grave sin signos de alarma y de niños sanos en el grupo de control requerido para alcanzar un alfa unidireccional de 0,05 con un poder de 0,80 , fue de 44 en cada grupo, asumiendo que el $36,0 \%$ de los expuestos y el $11,0 \%$ de los controles podrían desarrollar algún trastorno funcional gastrointestinal. Se comparó el número de niños que reportaron alguno de dichos trastornos durante el seguimiento en ambos grupos y se calculó el riesgo relativo. La significación estadística de la diferencia entre los grupos, se evaluó mediante la prueba de ji al cuadrado y la exacta de Fisher.

Según la Resolución 8430 del 4 de octubre de 1993 del Ministerio de Salud colombiano, este estudio se clasificó como sin riesgo. El estudio fue aprobado por el Comité de Ética de la Universidad del Valle y la rectoría de la institución educativa pública participante.

\section{Resultados}

\section{Características generales}

Se incluyeron 135 niños cuya edad promedio fue de 10,7 $\pm 1,9$ años (rango de 8 a 14 años), 69 eran de sexo masculino, el $37 \%$ presentaba malnutrición y el 9,6\% tenía una talla alterada. Del total, 19,3\% sufrieron algún trastorno funcional gastrointestinal, como estreñimiento funcional (9,6\%), síndrome de intestino irritable $(3,7 \%)$, síndrome de dolor abdominal funcional $(2,2$ $\%$ ), dolor abdominal funcional más dispepsia funcional (1,5\%) o migraña abdominal $0,7 \%$ ). No hubo diferencias significativas entre los 73 niños con dengue no grave y los 62 niños sanos del grupo de control (cuadro 1).

\section{Seguimiento de los trastornos funcionales gastrointestinales}

Después del episodio de dengue no grave, el riesgo de presentar algún trastorno funcional gastrointestinal fue mayor: 1,37 veces a los 3 meses $\left(\mathrm{IC}_{95 \%} 0,37-4,98\right), 1,13$ veces a los 6 meses $\left(\mathrm{IC}_{95 \%} 0,30-3,97\right), 1,27$ veces a los 9 meses $\left(\mathrm{IC}_{95 \%} 0,32-4,71\right)$ y 1,84 veces a los 12 meses, $\left(\mathrm{IC}_{95 \%} 0,27-\right.$ $13,77)$. No obstante, no hubo diferencias significativas ( $p>0,05)$, incluso al analizar dichos trastornos en los niños expuestos con dengue no grave (cuadro 2) y en los niños sanos de control (cuadro 3).

\section{Dolor abdominal relacionado con los trastornos funcionales gastrointestinales}

Al analizar el subgrupo de los niños con dolor abdominal relacionado con estos trastornos (dispepsia funcional, síndrome de intestino irritable, migraña abdominal, dolor abdominal funcional y síndrome de dolor abdominal funcional) tanto en el grupo de expuestos como en el de control, no hubo diferencias significativas en cuanto a la presencia de tales trastornos a los 3 meses $(p=0,469), 6$ meses $(p=0,398), 9$ meses $(p=0,406)$ y 12 meses $(p=0,459)$ de seguimiento (figura 1$)$. 
Cuadro 1. Características generales de 135 niños, 73 con dengue no grave sin signos de alarma y 62 controles sanos

\begin{tabular}{|c|c|c|c|}
\hline & $\begin{array}{c}\text { Expuestos } \\
(n=73) \\
n \quad \%\end{array}$ & $\begin{array}{c}\text { Controles } \\
(n=62) \\
n \%\end{array}$ & $\mathbf{P}$ \\
\hline Edad (años) & $10,6 \pm 1,9$ & $10,8 \pm 2,0$ & 0,693 \\
\hline \multicolumn{4}{|l|}{ Sexo } \\
\hline $\begin{array}{l}\text { Femenino } \\
\text { Masculino }\end{array}$ & $\begin{array}{l}33(45,2) \\
40(54,8)\end{array}$ & $\begin{array}{l}33(53,2) \\
29(46,8)\end{array}$ & 0,225 \\
\hline \multicolumn{4}{|l|}{ Estado nutricional } \\
\hline \multicolumn{4}{|l|}{ Según índice de masa corporal } \\
\hline $\begin{array}{l}\text { Eutrófico } \\
\text { Malnutrido }\end{array}$ & $\begin{array}{l}46(63,0) \\
27(37,0)\end{array}$ & $\begin{array}{l}39(62,9) \\
23(37,1)\end{array}$ & 0,565 \\
\hline \multicolumn{4}{|l|}{ Según talla para la edad } \\
\hline $\begin{array}{l}\text { Eutrófico } \\
\text { Talla alterada }\end{array}$ & $\begin{array}{l}52(71,2) \\
21(28,8)\end{array}$ & $\begin{array}{l}48(77,4) \\
14(22,6)\end{array}$ & 0,268 \\
\hline \multicolumn{4}{|l|}{ Trastornos funcionales gastrointestinales } \\
\hline $\begin{array}{l}\text { No } \\
\text { Sí }\end{array}$ & $\begin{array}{l}56(76,7) \\
17(23,3)\end{array}$ & $\begin{array}{r}53(85,5) \\
9(14,5)\end{array}$ & 0,142 \\
\hline Dolor abdominal relacionado con TFG & $6(8,3)$ & $7(11,2)$ & 0,475 \\
\hline Dispepsia funcional & $1(1,4)$ & $1(1,6)$ & 0,739 \\
\hline Síndrome de intestino irritable & $2(2,7)$ & $3(4,8)$ & 0,483 \\
\hline Migraña abdominal & $1(1,4)$ & $0 \quad(0,0)$ & 0,518 \\
\hline Dolor abdominal funcional & $1(1,4)$ & $1(1,6)$ & 0,739 \\
\hline Síndrome de dolor abdominal funcional & $1(1,4)$ & $2(3,2)$ & 0,486 \\
\hline Estreñimiento e incontinencia & $11(15,0)$ & $2(3,3)$ & 0,021 \\
\hline Estreñimiento funcional & $11(15,0)$ & $2(3,3)$ & 0,021 \\
\hline
\end{tabular}

TFG: trastornos funcionales gastrointestinales

Cuadro 2. Trastornos funcionales gastrointestinales en el grupo de niños expuestos a los 3, 6, 9 y 12 meses de presentar un episodio de dengue no grave sin signos de alarma

\begin{tabular}{|c|c|c|c|c|c|c|c|c|c|c|c|c|c|c|}
\hline & \multicolumn{2}{|c|}{$\begin{array}{c}\text { Día 0 } \\
(n=73)\end{array}$} & \multicolumn{3}{|c|}{$\begin{array}{c}3 \text { meses } \\
(n=47)\end{array}$} & \multicolumn{3}{|c|}{$\begin{array}{c}6 \text { meses } \\
(n=36)\end{array}$} & \multicolumn{3}{|c|}{$\begin{array}{c}9 \text { meses } \\
(n=33)\end{array}$} & \multicolumn{3}{|c|}{$\begin{array}{c}12 \text { meses } \\
(n=25)\end{array}$} \\
\hline & $\mathbf{n}$ & $\%$ & $\mathbf{n}$ & $\%$ & $\mathbf{p}$ & $\mathbf{n}$ & $\%$ & $\mathbf{P}$ & $\mathbf{n}$ & $\%$ & $\mathbf{p}$ & $\mathbf{n}$ & $\%$ & p \\
\hline \multicolumn{15}{|l|}{ Trastornos funciones gastrointestinales } \\
\hline No & \multicolumn{2}{|c|}{$56(76,7)$} & \multicolumn{2}{|c|}{$40(85,1)$} & 0,188 & \multicolumn{2}{|c|}{$30(83,3)$} & 0,297 & \multicolumn{2}{|c|}{$27(81,8)$} & 0,375 & \multicolumn{2}{|c|}{$21(84,0)$} & 0,322 \\
\hline Sí & \multicolumn{2}{|c|}{$17(23,3)$} & \multicolumn{2}{|c|}{$7(14,9)$} & & \multicolumn{2}{|c|}{$6(16,7)$} & & \multicolumn{2}{|c|}{$6(18,2)$} & & \multicolumn{2}{|c|}{$4(16,0)$} & \\
\hline Vómito y aerofagia & 0 & $(0,0)$ & 1 & $(2,1)$ & 0,423 & & & & 1 & $(3,0)$ & 0,333 & 1 & $(4,0)$ & 0,282 \\
\hline Aerofagia & 0 & $(0,0)$ & & & & & & & & & & 1 & $(4,0)$ & 0,282 \\
\hline Síndrome de rumiación del adolescente & 0 & $(0,0)$ & 1 & $(2,1)$ & 0,423 & & & & 1 & $(3,0)$ & 0,333 & & & \\
\hline Dolor abdominal relacionado con TFG & 6 & $(8,3)$ & 4 & $(8,5)$ & 0,598 & 2 & $(5,6)$ & 0,446 & 0 & $(0,0)$ & 0,106 & 1 & $(4,0)$ & 0,407 \\
\hline Dispepsia funcional & 1 & $(1,4)$ & 1 & $(2,1)$ & 0,664 & 0 & $(0,0)$ & 0,655 & 0 & $(0,0)$ & 0,679 & 0 & $(0,0)$ & 0,731 \\
\hline Síndrome de intestino irritable & 2 & $(2,7)$ & 3 & $(6,4)$ & 0,360 & 1 & $(2,8)$ & 0,723 & 0 & $(0,0)$ & 0,463 & 0 & $(0,0)$ & 0,537 \\
\hline Migraña abdominal & 1 & $(1,4)$ & 0 & $(0,0)$ & 0,588 & 0 & $(0,0)$ & 0,655 & 0 & $(0,0)$ & 0,679 & 0 & $(0,0)$ & 0,731 \\
\hline Dolor abdominal funcional & 1 & $(1,4)$ & 0 & $(0,0)$ & 0,588 & 0 & $(0,0)$ & 0,655 & 0 & $(0,0)$ & 0,679 & 0 & $(0,0)$ & 0,731 \\
\hline Síndrome de dolor abdominal funcional & 1 & $(1,4)$ & 0 & $(0,0)$ & 0,588 & 1 & $(2,8)$ & 0,583 & 0 & $(0,0)$ & 0,679 & 1 & $(4,0)$ & 0,482 \\
\hline Estreñimiento e incontinencia & 11 & $(15,1)$ & 2 & $(4,3)$ & 0,059 & & $(11,1)$ & 0,381 & & $(15,2)$ & 0,585 & 2 & $(8,0)$ & 0,297 \\
\hline Estreñimiento funcional & 11 & $(15,1)$ & 2 & $(4,3)$ & 0,059 & 3 & $(8,3)$ & 0,251 & & $(15,2)$ & 0,585 & 2 & $(8,0)$ & 0,297 \\
\hline Incontinencia fecal no retentiva & 0 & $(0,0)$ & & & & 1 & $(2,8)$ & 0,356 & & & & & & \\
\hline
\end{tabular}


Cuadro 3. Trastornos funcionales gastrointestinales (TFG) en el grupo de control a los 3, 6, 9 y 12 meses de seguimiento

\begin{tabular}{|c|c|c|c|c|c|c|c|c|c|c|c|c|c|c|}
\hline & \multicolumn{2}{|c|}{$\begin{array}{c}\text { Día } 0 \\
(n=62)\end{array}$} & \multicolumn{3}{|c|}{$\begin{array}{c}3 \text { meses } \\
(n=62)\end{array}$} & \multicolumn{3}{|c|}{$\begin{array}{c}6 \text { meses } \\
(n=60)\end{array}$} & \multicolumn{3}{|c|}{$\begin{array}{c}9 \text { meses } \\
(n=54)\end{array}$} & \multicolumn{3}{|c|}{$\begin{array}{c}12 \text { meses } \\
(n=32)\end{array}$} \\
\hline & $\mathbf{n}$ & $\%$ & $\mathbf{n}$ & $\%$ & $\mathbf{p}$ & $\mathbf{n}$ & $\%$ & $\mathbf{p}$ & $\mathbf{n}$ & $\%$ & $\mathbf{p}$ & $\mathbf{n}$ & $\%$ & $\mathbf{p}$ \\
\hline \multicolumn{15}{|l|}{ Trastornos funciones gastrointestinales } \\
\hline No & \multicolumn{2}{|c|}{$53(85,5)$} & \multicolumn{2}{|c|}{$55(88,7)$} & 0,395 & \multicolumn{2}{|c|}{$51(85,0)$} & 0,571 & \multirow{2}{*}{\multicolumn{2}{|c|}{$\begin{array}{r}46(85,2) \\
8(14,8)\end{array}$}} & 0,584 & \multicolumn{2}{|c|}{$29(90,6)$} & 0,361 \\
\hline Sí & & $(14,5)$ & & $(11,3)$ & & & $15,0)$ & & & & & 3 & $(9,4)$ & \\
\hline Vómito y aerofagia & 0 & $(0,0)$ & & & & 1 & $(1,7)$ & 0,495 & 1 & $(1,9)$ & 0,470 & & & \\
\hline Aerofagia & 0 & $(0,0)$ & & & & 1 & $(1,7)$ & 0,495 & 1 & $(1,9)$ & 0,470 & & & \\
\hline Dolor abdominal relacionado con TFG & & $(11,2)$ & 4 & $(6,4)$ & 0,274 & & $10,0)$ & 0,540 & 2 & $(3,7)$ & 0,147 & 3 & $(9,4)$ & 0,518 \\
\hline Dispepsia funcional & 1 & $(1,6)$ & 0 & $(0,0)$ & 0,495 & 0 & $(0,0)$ & 0,514 & 0 & $(0,0)$ & 0,540 & 0 & $(0,0)$ & 0,651 \\
\hline Síndrome de intestino irritable & 3 & $(4,8)$ & 2 & $(3,2)$ & 0,492 & 4 & $(6,7)$ & 0,490 & 0 & $(0,0)$ & 0,161 & 1 & $(3,1)$ & 0,565 \\
\hline Migraña abdominal & 0 & $(0,0)$ & 1 & $(1,6)$ & 0,514 & & & & & & & & & \\
\hline Dolor abdominal funcional & 1 & $(1,6)$ & 1 & $(1,6)$ & 0,743 & 0 & $(0,0)$ & 0,514 & 2 & $(3,7)$ & 0,455 & 2 & $(6,3)$ & 0,300 \\
\hline Síndrome de dolor abdominal funcional & 2 & $(3,2)$ & 0 & $(0,0)$ & 0,248 & 2 & $(3,3)$ & 0,677 & 0 & $(0,0)$ & 0,294 & 0 & $(0,0)$ & 0,426 \\
\hline Estreñimiento e incontinencia & 2 & $(3,2)$ & 3 & $(4,8)$ & 0,525 & 2 & $(3,3)$ & 0,677 & 5 & $(9,3)$ & 0,189 & 0 & $(0,0)$ & 0,426 \\
\hline Estreñimiento funcional & 2 & $(3,2)$ & 3 & $(4,8)$ & 0,525 & 2 & $(3,3)$ & 0,677 & 5 & $(9,3)$ & 0,189 & 0 & $(0,0)$ & 0,426 \\
\hline
\end{tabular}

TFG: trastornos funcionales gastrointestinales

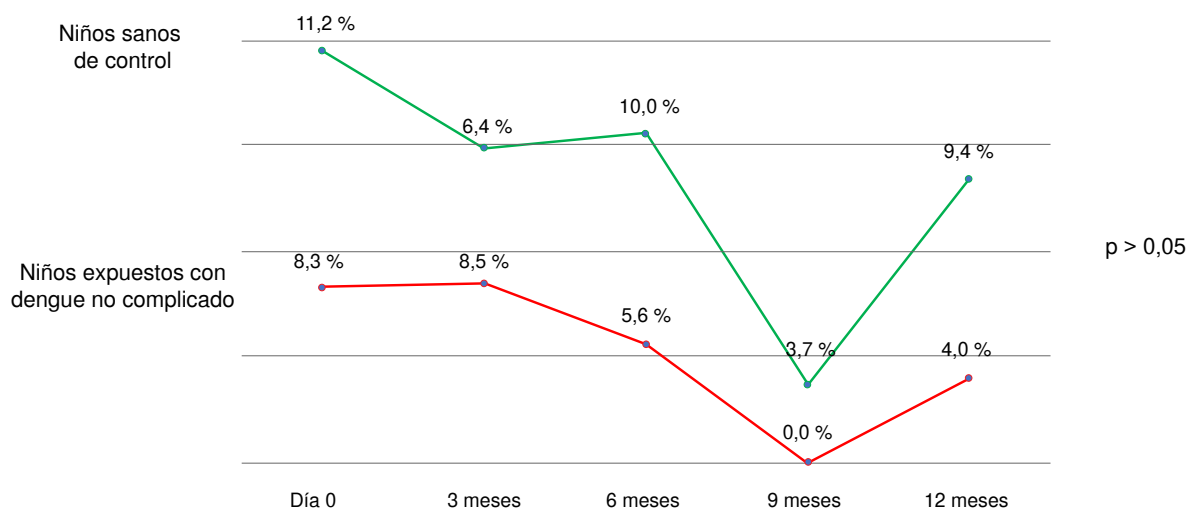

Figura 1. Seguimiento del dolor abdominal relacionado con trastornos funcionales gastrointestinales en niños expuestos con dengue no grave y en niños sanos (grupo de control)

\section{Discusión}

Según los resultados obtenidos, no se presentan trastornos funcionales gastrointestinales después de la infección por dengue no grave, ni siquiera cuando se considera el dolor abdominal relacionado con dichos trastornos o el síndrome de intestino irritable.

Los datos de este estudio son contrarios a los reportados por Saps, et al. (4) en casos de trastornos funcionales gastrointestinales debidos a diarrea bacteriana, y por Pensabene, et al. (5), en los causados por diarrea de etiología múltiple. En cambio, coinciden con otro estudio registrado por Saps, et al. (6) sobre trastornos con diarrea causada por rotavirus. En cuanto a la enfermedad diarreica aguda bacteriana, en los resultados de Saps, et al. (4) en una cohorte de 88 niños italianos y norteamericanos (46 de sexo masculino) entre los 3 y los 19 años de edad (edad media de 8,1 años), hubo 44 niños con coprocultivos positivos (54,0\% para Salmonella spp., 32,0 \% para Campylobacter spp.y 14,0 $\%$ para Shigella spp.) y 44 con coprocultivos negativos durante el seguimiento de seis meses para comprobar la presencia de trastornos funcionales gastrointestinales después de un episodio de diarrea aguda bacteriana. 
Los autores reportaron, asimismo, dolor abdominal en $36 \%$ de los participantes del grupo de expuestos y en $11 \%$ del grupo de control $(p<0,01)$. El principal trastorno, según los criterios de Roma II, fue el síndrome de intestino irritable $(87,0 \%)$ seguido de la dispepsia funcional $(24 \%)$.

En cuanto a la enfermedad diarreica aguda de etiología múltiple, en el estudio multicéntrico de Pensabene, et al. (5) en 64 niños italianos (36 de sexo masculino), entre los 4,1 y 14,1 años de edad (edad media: 5,3 años), en el que las muestras de heces de 32 niños fueron positivas (56,8\% para rotavirus, 30,0 \% para Salmonella spp., 6,6 \% para adenovirus, 3,3 \% para norovirus y $3,3 \%$ para Giardia lamblia) y 32 negativas, los autores reportaron que el riesgo de desarrollar algún trastorno funcional gastrointestinal después de un episodio de diarrea aguda en el primer mes fue 1,9 veces mayor $(p=0,02)$; a los 3 meses, 1,13 veces mayor $(p=0,003)$ y a los 6 meses, 1,9 veces mayor $(p=0,01)$, incluso cuando se analizó la presencia de dolor abdominal relacionado con los trastornos $(R R=1,7 ; p=0,04)$ según los criterios de Roma III.

Por último, en el estudio de cohortes sobre enfermedad diarreica aguda de origen viral (4) en 88 niños italianos y norteamericanos (46 de sexo masculino) entre los 4 y los 18 años de edad (edad media: 5,3 años), 44 de los cuales fueron positivos para rotavirus en heces y 44 negativos, en el seguimiento de hasta 2 años después del episodio de diarrea aguda viral se reportaron trastornos funcionales gastrointestinales en el 16,0\% del grupo de expuestos y en el 7,0 \% del grupo de control, con presencia de dolor abdominal $(p=0,31)$; el principal trastorno fue el síndrome de intestino irritable seguido por el dolor abdominal funcional según los criterios de Roma II.

En el presente estudio no se determinó ningún factor de riesgo, como sexo, edad o estado nutricional, para desarrollar trastornos funcionales gastrointestinales, lo que concuerda con lo reportado por Saps, et al. (4) en niños con dichos trastornos por diarrea bacteriana y con los resultados de Pensabene, et al. (5) en niños con tales trastornos por diarrea de diferentes etiologías.

En el presente estudio tampoco hubo incremento de la prevalencia de dichos trastornos a los 3, 6, 9 y 12 meses de seguimiento, ni en los niños expuestos ni en los sanos, lo que contradice los hallazgos de Saps, et al. (4) durante seguimiento de 6 meses de niños con trastornos funcionales gastrointestinales secundarios a diarrea bacteriana, pues los autores reportaron un riesgo relativo de $3,2\left(\mathrm{IC}_{95 \%} 1,284-7,975 ; \mathrm{p}>0,05\right)$ de presentar alguno de estos trastornos después de la enfermedad diarreica aguda bacteriana, así como los de Pensabene, et al. (5), quienes a los 6 meses de seguimiento reportaron dichos trastornos en $45,0 \%$ de los participantes con enfermedad diarreica aguda de origen viral y en $54,5 \%$ de aquellos con la de origen bacteriano.

En el presente estudio, la prevalencia más alta de dolor abdominal relacionado con estos trastornos durante el seguimiento del grupo de expuestos, fue de $8,5 \%$ a los 3 meses, datos por debajo de los registrados por Saps, et al. (4), 36,0 \% en diarrea bacteriana, y por Pensabene, et al. (5), $28,1 \%$ en diarrea de etiología múltiple. La prevalencia más alta en nuestro grupo de control fue de $10,0 \%$ a los 6 meses, datos similares a los reportados por Saps, et al. (4) para dichos trastornos después de un episodio de diarrea bacteriana $(11,0 \%)$ y algo superiores a los reportados por Pensabene, et al. (5) después de uno de diarrea de etiología múltiple (6,2\%). 
Saps, et al. (4) sugieren que bacterias como Salmonella, Shigella y Campylobacter (todas asociadas con enfermedad diarreica aguda), frecuentemente se relacionan con el síndrome de intestino irritable después de una infección en niños, y que una alta proporción de niños con esta enfermedad desarrollan trastornos funcionales gastrointestinales con síntomas que pueden persistir por años después del episodio infeccioso. Estos mismos autores no encontraron una relación significativa entre la enfermedad diarreica aguda por rotavirus y dichos trastornos en el seguimiento a largo plazo después de un episodio de diarrea viral (6). Por último, Pensabene, et al. (5), en su estudio multicéntrico prospectivo de cohortes, sugieren que estos trastornos después de un episodio infeccioso son una verdadera enfermedad en los niños, y concluyen que hubo un incremento significativo del dolor abdominal relacionado con tales trastornos después de un episodio diarreico agudo en niños en los meses 1, 3 y 6 de seguimiento.

El uso de una población diversa, el cálculo a priori del tamaño de la muestra, el grupo sano de control, la confirmación clínica y paraclínica en los niños expuestos, la validación previa y el cuestionario de los criterios de Roma III estandarizado en español para detectar trastornos funcionales gastrointestinales, constituyen las fortalezas de esta investigación. Otra característica favorable en este estudio para su validez interna fue la prevalencia de dichos trastornos en el grupo de control (14,5\%), cuyas cuatro principales causas (estreñimiento funcional, síndrome de intestino irritable, dolor abdominal funcional y síndrome de dolor abdominal funcional) son similares a las reportadas previamente en estudios epidemiológicos en niños colombianos y latinoamericanos realizados por el Functional International Digestive Epidemiological Research Survey (FINDERS) (11-15).

Entre las limitaciones del estudio debe mencionarse que los trastornos funcionales gastrointestinales no se diagnosticaron clínicamente, tampoco se hizo la serotipificación del virus del dengue y pudo darse un sesgo de selección debido al corto tiempo de duración del estudio.

En conclusión, los hallazgos del presente estudio no indican que los trastornos funcionales gastrointestinales se presenten después de un episodio de dengue no grave en niños. Se necesitan más estudios prospectivos que exploren el papel de las infecciones virales en el desarrollo de estos trastornos después de la infección, así como para caracterizar la población pediátrica con riesgo alto de desarrollarlos. Estos estudios deben investigar los factores de riesgo que predisponen a los niños, con el fin de proponer estrategias de intervención orientadas a reducirlos.

Se recomienda realizar un nuevo estudio basado en los actuales criterios de Roma IV que incluyen, entre otros, las náuseas y el vómito funcional, los subtipos de dispepsia funcional (síndrome de malestar posprandial y síndrome de dolor epigástrico) y los subtipos del síndrome de intestino irritable (con estreñimiento, con diarrea, mixto y sin clasificar) (3). Asimismo, se recomienda hacer un estudio en las diferentes regiones del país e, incluso, en varios países durante la época de mayor presencia de casos de dengue y empleando preferentemente la serotipificación viral, para comparar posibles diferencias epidemiológicas.

\section{Referencias}

1. Saps M, Nichols-Vinueza DX, Rosen JM, Velasco-Benítez CA. Prevalence of functional gastrointestinal disorders in Colombian school children. J Pediatr. 2014;164:542-5. https://doi.org/10.1016/j.jpeds.2013.10.088 
2. Saps M, Moreno-Gómez JE, Ramírez-Hernández CR, Rosen JM, Velasco-Benítez CA. A nationwide study on the prevalence of functional gastrointestinal disorders in school children. Bol Med Hosp Infant Mex. 2017;76:407-12. https://doi.org/10.1016/j.bmhimx.2017.05.005

3. Hyams JS, Di Lorenzo C, Saps M, Shulman RJ, Staiano A, van Tilburg M. Childhood functional gastrointestinal disorders: Child/adolescent. Gastroenterology. 2016;150:1456-68. https://doi.org/10.1053/j.gastro.2016.02.015

4. Saps M, Pensabene L, Di Martino L, Staiano AM, Wechsler J, Zheng X, et al. Post-infectious functional gastrointestinal disorders in children. J Pediatr. 2008;152:812-56. https://doi.org/10.1016/j.jpeds.2007.11.042

5. Pensabene L, Talarico V, Concolino D, Ciliberto D, Campanozzi A, Gentile T, et al. Postinfectious functional gastrointestinal disorders in children: A multicenter prospective study. J Pediatr. 2015;166: 903-7. https://doi.org/10.1016/j.jpeds.2014.12.050

6. Saps M, Pensabene L, Turco R, Staiano A, Cupuro D, Di Lorenzo C. Rotavirus gastroenteritis: Precursor of functional gastrointestinal disorders? J Pediatr Gastroenterol Nutr. 2009;49:580-3. https://doi.org/10.1097/MPG.0b013e31819bcbd2

7. Castrillón JC, Castaño JC, Urcuqui S. Dengue en Colombia: diez años de evolución. Rev Chilena Infectol. 2015;32:142-9. httpa://doi.org/10.4067/S0716-10182015000300002

8. Hernández M, Arboleda D, Arce S, Benavides A, Tejada PA, Ramírez SV, et al. Metodología para la elaboración de canales endémicos y tendencia de la notificación del dengue, Valle del Cauca, Colombia, 2009-2013. Biomédica. 2016;36(Supl.2):98-107. https://doi.org/10.7705/biomedica.v36i0.2934

9. Verhagen LM, de Groot R. Dengue in children. J Infect. 2014;69(Supl.1):S77-86. https://doi.org/10.1016/j.jinf.2014.07.020

10. Velasco-Benítez CA, Ortiz-Rivera CJ. ¿El antecedente de dengue está asociado a la presencia de desórdenes gastrointestinales funcionales en niños? Infectio. 2019;23:161-6. https://doi.org/10.22354/in.v23i2.773

11. Jativa E, Velasco-Benítez CA, Koppen IJN, Jativa Z, Saps M. Prevalence of functional gastrointestinal disorders in school children in Ecuador. J Pediatr Gastroenterol Nutr. 2016;63:25-8. https://doi.org/10.1097/MPG.0000000000001108

12. Dhroove G, Saps M, García-Bueno C, Leyva-Jiménez A, Rodríguez-Reynosa LL, VelascoBenítez CA. Prevalencia de trastornos gastrointestinales funcionales en escolares mexicanos. Rev Gastroenterol Mex. 2017;82:13-8. https://doi.org/10.1016/j.rgmx.2016.05.003

13. Zablah R, Velasco-Benítez CA, Merlos I, Bonilla S, Saps M. Prevalencia de trastornos funcionales gastrointestinales en niños en edad escolar en El Salvador. Rev Gastroenterol Mex. 2015;80:186-91. https://doi.org/10.1016/j.rgmx.2015.03.008

14. Mejía M, Velasco-Benítez CA, Díaz J. La prevalencia y las posibles asociaciones de los desórdenes gastrointestinales funcionales en escolares y adolescentes de colegios privados de Managua, Nicaragua. Acta Gastroenterol Latinoam. 2017;47:163-8.

15. Lu PL, Saps M, Chanis RA, Velasco-Benítez CA. The prevalence of functional gastrointestinal disorders in children in Panama: A school-based study. Acta Pædiatr. 2016;105:e232-6. https://doi.org/10.1111/apa.13379

16. de Onis M, Onyango AW, Borghi E, Siyam A, Nishida C, Siekmann J. Development of a WHO growth reference for school-aged children and adolescents. BWHO. 2007; 85: 660-7. 